\title{
SYNTHESIS AND SPECTRAL CHARACTERIZATION OF SOME NOVEL $N$-ACYL-PIPERIDIN-4-ONES
}

\author{
K. Selvaraju ${ }^{1^{*}}$ and A. Manimekalai ${ }^{2}$ \\ ${ }^{1}$ Department of Chemistry, Sri Sairam Engineering College, Chennai-600 044, Tamil Nadu, India \\ ${ }^{2}$ Department of Chemistry, Annamalai University, Annamalainagar, Chidambaram-608 002 \\ Tamil Nadu, India \\ *E-mail: jkselvaraju@gmail.com
}

\begin{abstract}
A collection of novel $N$-acyl-t(3)-ethyl-r(2),c(6)-di(2'-furyl)piperidin-4-ones 1-4 are synthesized, characterized by melting point, one-dimensional NMR $\left({ }^{1} \mathrm{H}\right.$ and $\left.{ }^{13} \mathrm{C}\right)$ and two-dimensional NOESY and ${ }^{1} \mathrm{H}_{-}{ }^{13} \mathrm{C}$ COSY spectrum. The spectra reveal the presence of two isomeric forms i.e., $\mathrm{E}$ and $\mathrm{Z}$ isomers for 1-4. The effect of varying the substituents at nitrogen on the ${ }^{1} \mathrm{H}$ and ${ }^{13} \mathrm{C}$ chemical shifts have been analyzed in detail.
\end{abstract}

Keywords: NMR spectra, ${ }^{1} \mathrm{H}$ NMR, ${ }^{13} \mathrm{C}$ NMR, $N$-acyl-piperidin-4-ones

(C) RASĀYAN. All rights reserved

\section{INTRODUCTION}

Among the family of heterocyclic compounds, the nitrogen-containing six-membered heterocycles, the piperidine structural was the most dominant and very prevalent element in nature and often found to be naturally occurring bioactive compounds such as alkaloids ${ }^{1}$. Piperidin-3-one derivatives are used as precursors for the synthesis of antimalarial agents Febrifugine and Isofebrifugine ${ }^{2}$. Piperidin-4-ones mostly display varied and potent biological properties such as Antiviral, Antitumour ${ }^{3}$, Analgesic ${ }^{4}$, Antimicrobial $^{5}$, Fungicidal ${ }^{5}$, Herbicidal, Insecticidal, Antihistaminic, Anti-inflammatory and Anticancer. CNS stimulant and recent reports suggest that compounds containing piperidin-4-one moiety elicit excellent activity when aromatic substitutions are present at 2- and/or 6-positions.

Mannich reaction is one of the multicomponent reactions for the carbon-carbon and carbon-hetero atom sequential bond formation. Mannich type condensation involving aromatic aldehydes, ammonium acetate and ketones having two active methylene groups, resulting in the formation of 2,6-diarylpiperidin-4-ones, was first reported by Noller and Baliah ${ }^{6}$. In the course of developing biologically active molecules, we have recently reported the synthesis and conformational studies of $N$-acyl-t(3)-methyl -2,6difurylpiperidin-4-ones ${ }^{7}$ and $\mathrm{N}$-acyl-t(3)-isopropyl-2,6-difurylpiperidin-4-one oxime derivatives ${ }^{8}$. In the present study $N$-acyl-t(3)-ethyl-r(2),c(6)-di(2'-furyl)piperidin-4-ones (1-4) synthesized and analyzed by ${ }^{1} \mathrm{H},{ }^{13} \mathrm{C},{ }^{1} \mathrm{H}-{ }^{13} \mathrm{C}$ COSY and NOESY spectrum.

\section{EXPERIMENTAL}

${ }^{1} \mathrm{H}$ and ${ }^{13} \mathrm{C}$ NMR spectra were recorded on a Bruker AMX 400 NMR spectrometer operating at 400 and $100.6 \mathrm{MHz}$ for ${ }^{1} \mathrm{H}$ and ${ }^{13} \mathrm{C}$ respectively. The NOESY and ${ }^{1} \mathrm{H}-{ }^{13} \mathrm{C}$ COSY spectra were recorded on a Bruker DRX 500 NMR spectrophotometer.

The starting compound $t(3)$-ethyl-r(2),c(6)-di(2'-furyl)piperidin-4-one was prepared by the general procedure reported by Noller and Baliah ${ }^{6}$. A mixture of ammonium acetate $(0.05 \mathrm{~mol})$, furfuraldehyde $(0.1$ $\mathrm{mol})$ and pentan-2-one $(0.05 \mathrm{~mol})$ in distilled ethanol was heated first to boiling and then stirred under the cold condition for $1 \mathrm{hr}$. Then the reaction mass was added $200 \mathrm{~mL}$ ether and $20 \mathrm{~mL}$ of concentrated hydrochloric acid. The hydrochloride salt was precipitated out and filtered the salt and washed with $40 \mathrm{~mL}$ mixture of ethanol and ether (1:1). The base was liberated by addition of aqueous ammonia followed by dilution with chilled water. The compound was recrystallized from benzene- 
petroleum ether mixture. Yield: $70 \%$ m.p. $40^{\circ} \mathrm{C}$.

The $N$-formyl derivative 1 was prepared from parent piperidin-4-one by the procedure reported in the literature ${ }^{9}$. Charged $50 \mathrm{~mL}$ of acetic anhydride into a $250 \mathrm{ml} 4$ neck RB flask followed by cooled the contents to $0-5^{\circ} \mathrm{C}$. Then added $25 \mathrm{~mL}$ of formic acid over a period of 30 minutes. Raised the temperature to $50-60^{\circ} \mathrm{C}$ and maintained for $1 \mathrm{hr}$. After maintenance reduced the temperature to $0-5^{\circ} \mathrm{C}$. Then slowly added the solution of parent piperidin-4-one (dissolved in benzene) at $0-5{ }^{\circ} \mathrm{C}$, then increased the temperature to $25-30^{\circ} \mathrm{C}$ and maintained the contents till the completion of the reaction. After completion of the reaction, the reaction mass washed with $10 \% \mathrm{Na}_{2} \mathrm{CO}_{3}$ solution. Finally, the product isolated from the organic layer. Yield: $70 \%$, m.p. $95^{\circ} \mathrm{C}$.

The other $N$-acyl derivatives $\mathbf{2 - 4}$ were prepared as per the procedure reported in the literature ${ }^{10}$. Charged benzene into a $250 \mathrm{ml} 4$ neck RB flask, then added 1.0 mole of parent piperidin-4-one and stirred the reaction mass to 5 -10 minutes. Then slowly added 1.0 mole of acetic anhydride/propanoic anhydride/benzoyl chloride followed by 0.5 moles of triethyl amine at $25-30{ }^{\circ} \mathrm{C}$. Then raised to reflux temperature and maintained the reaction mass up to completion of the reaction. After completion of the reaction, the reaction mass washed with $10 \% \mathrm{NaCl}$ solution. Then the product is isolated from the organic layer. 2: Yield: 50-60\%, m.p. $80^{\circ} \mathrm{C}$ 3: Yield: $40-50 \%$; m.p. $70^{\circ} \mathrm{C}, 4$ : Yield: $70 \%$; m.p. $125^{\circ} \mathrm{C}$.

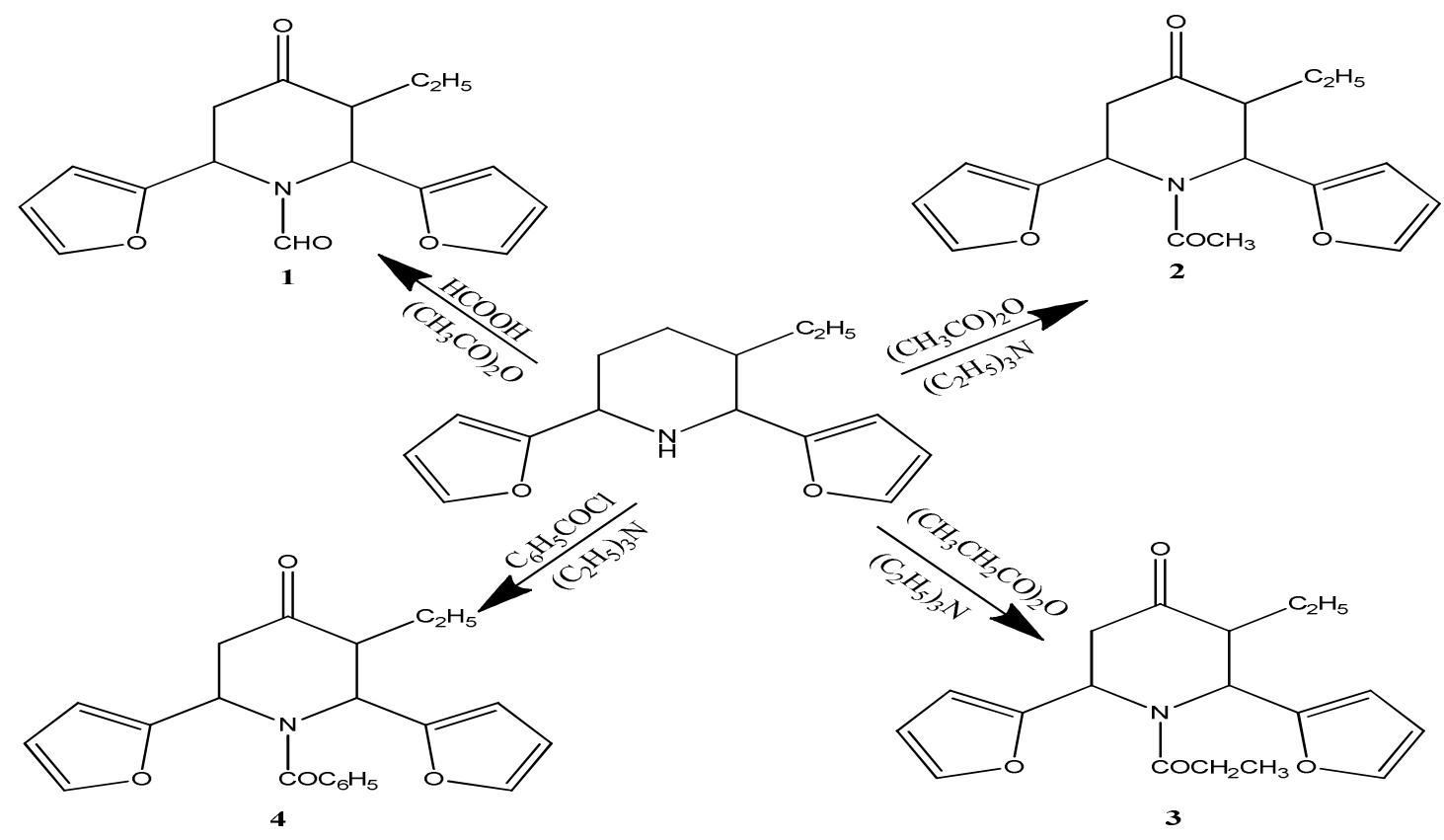

Scheme-1

\section{RESULTS AND DISCUSSION}

The $\quad N$-formyl-t(3)-ethyl-r(2), $c(6)$-di(2'-furyl)piperidin-4-one $\quad \mathbf{1}, \quad N$-acetyl-t(3)-ethyl-r(2), $c(6)$-di(2'furayl)piperidin-4-one $\quad 2, \quad N$-propanoyl-t(3)-ethyl-r(2),c(6)-di(2'-furyl)piperidin-4-one $\mathbf{3}$ and $N$ benzoyl-t(3)-ethyl-r(2),c(6)-di(2'-furyl)piperidin-4-one 4 were synthesized and analyzed by ${ }^{1} \mathrm{H},{ }^{13} \mathrm{C}$, NOESY and ${ }^{1} \mathrm{H}_{-}^{13} \mathrm{C}$ COSY spectrum. At room temperature, the signals of the spectrum are very broad. The well-resolved signals are obtained at lower temperatures only $\left(+15,0,-15\right.$ and $\left.\quad-30^{\circ} \mathrm{C}\right)$. The ${ }^{1} \mathrm{H}$ and ${ }^{13} \mathrm{C}$ NMR spectra reveal the presence of two isomers obtained at lower temperatures. The two isomers in 1-4 suggest the presence of restricted rotation around $\mathrm{N}-\mathrm{C}$ bonds and establishment of equilibrium between two rotamers with the coplanar orientation of acyl group. The two isomers are labeled as $Z$ [carbonyl group is syn to ethyl group at $\mathrm{C}(3)$ ] and $E$ [carbonyl group is anti to ethyl group at $\mathrm{C}(3)$ ] isomers (Figure - 1). 
RASĀYAN J. Chem.

Vol. 10 | No. 1 |25-31 | January - March | 2017

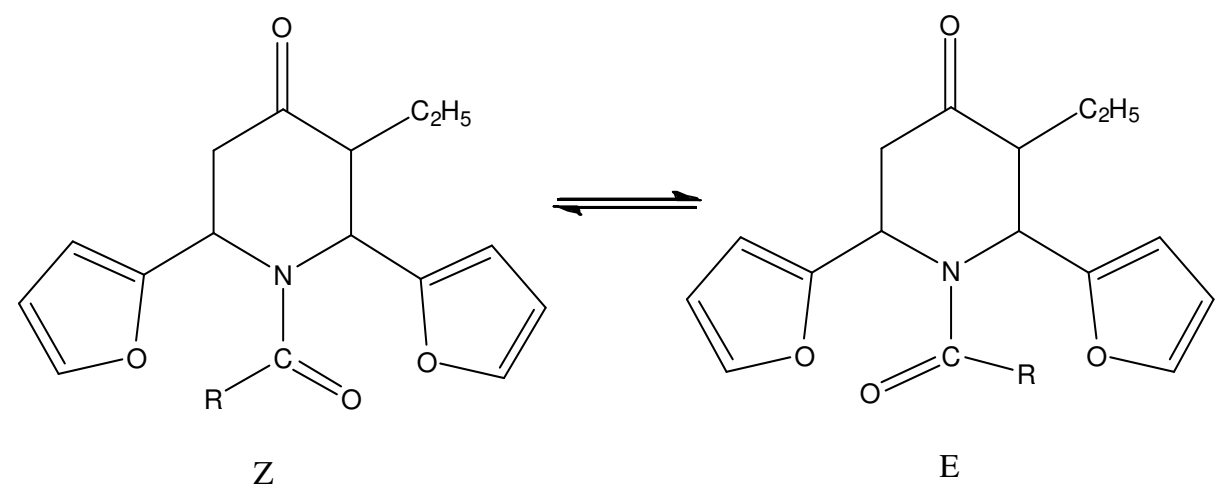

Fig.-1

The two isomers can be easily separated based on the height of the peaks. The proton signals of two isomers were confirmed by NOESY spectrum at $-30^{\circ} \mathrm{C}$ for 1 and ${ }^{1} \mathrm{H}-{ }^{13} \mathrm{C} \mathrm{COSY}$ spectrum at $-30^{\circ} \mathrm{C}$ for 2-4. Table-1 displayed the ${ }^{1} \mathrm{H}$ NMR values and the coupling constant values for $\mathbf{1 - 4}$. The ${ }^{13} \mathrm{C}$ NMR values of 1-4 are displayed in Table-2. The ${ }^{1} \mathrm{H}$ and ${ }^{13} \mathrm{C}$ NMR values of parent $t(3)$-ethyl-r(2),c(6)-di(2'furyl)piperidin-4-one are reported in literature ${ }^{11}$.

Table-1: ${ }^{1} \mathrm{H}$ NMR values (ppm) of $N$-acyl-3-ethylpiperidin-4-ones (1-4)

\begin{tabular}{|c|c|c|c|c|c|c|c|}
\hline Compd. & $\mathrm{H}(2)$ & $\mathrm{H}(3)$ & $\mathrm{H}(5)$ & $\mathrm{H}(6)$ & $\begin{array}{l}\text { Alkyl } \\
\text { protons }\end{array}$ & $\begin{array}{l}\text { Acyl } \\
\text { protons }\end{array}$ & Aromatic protons \\
\hline $\mathrm{E}$ & $\begin{array}{l}4.90 \\
(\mathrm{~s})\end{array}$ & $\begin{array}{l}2.88- \\
2.91\end{array}$ & $\begin{array}{l}2.88- \\
2.91\end{array}$ & $\begin{array}{l}6.11(\mathrm{t}, 5 \\
07 ; 10.1 \\
\left.4^{\mathrm{b}}\right)\end{array}$ & $\begin{array}{l}1.00(\mathrm{t}, \\
7.30) \\
\left(\mathrm{CH}_{2} \mathbf{C H}_{3}\right) \\
1.71-1.83 \\
\left(\mathbf{C H}_{2} \mathrm{CH}_{3}\right)\end{array}$ & 8.45 & \multirow[t]{2}{*}{$\begin{array}{l}5.91(2 \mathrm{H}), 5.95(2 \mathrm{H}), \\
6.04(2 \mathrm{H}), 6.08(2 \mathrm{H}), \\
7.04(2 \mathrm{H}), 7.11(2 \mathrm{H})\end{array}$} \\
\hline $\mathrm{Z}$ & $\begin{array}{l}5.93 \\
(d, 3.1 \\
9)\end{array}$ & $\begin{array}{l}2.88- \\
2.91\end{array}$ & $\begin{array}{l}2.92- \\
3.01\end{array}$ & $\begin{array}{l}5.23 \\
(\mathrm{~d}, 5.84)\end{array}$ & $\begin{array}{l}1.00(\mathrm{t}, \\
7.30) \\
\left(\mathrm{CH}_{2} \mathbf{C H}_{3}\right) \\
1.71-1.83 \\
\left(\mathbf{C H}_{2} \mathrm{CH}_{3}\right)\end{array}$ & 8.58 & \\
\hline $\mathrm{E}$ & $\begin{array}{l}5.25 \\
(\mathrm{~s})\end{array}$ & $\begin{array}{l}2.80- \\
2.87\end{array}$ & $\begin{array}{l}2.95 \\
\text { (dd, } \\
8.23,1 \\
5.27)\end{array}$ & $\begin{array}{l}6.45 \\
(d, 7.92)\end{array}$ & $\begin{array}{l}1.03(\mathrm{t}, 7.53) \\
\left.\left(\mathrm{CH}_{2} \mathbf{C H}\right)_{3}\right) \\
1.77-1.80 \\
\left(\mathbf{C H}_{2} \mathrm{CH}_{3}\right)\end{array}$ & 2.51 & \multirow{2}{*}{$\begin{array}{ll}5.84(1 \mathrm{H}), & 5.87(2 \mathrm{H}), \\
5.89(1 \mathrm{H}), & 6.03(2 \mathrm{H}), \\
6.07(1 \mathrm{H}), & 7.01(1 \mathrm{H}), \\
7.04(1 \mathrm{H}), & 7.08(1 \mathrm{H}), \\
7.12(1 \mathrm{H}) & \end{array}$} \\
\hline $\mathrm{Z}$ & $\begin{array}{l}6.27 \\
(\mathrm{~s})\end{array}$ & $\begin{array}{l}2.80- \\
2.87\end{array}$ & $\begin{array}{l}3.10 \\
\text { (dd, } \\
8.20,1 \\
5.54) \\
2.80- \\
2.87\end{array}$ & $\begin{array}{l}5.56 \\
(\mathrm{~d}, 7.88)\end{array}$ & $\begin{array}{l}1.00(\mathrm{t}, 7.54) \\
\left.\left(\mathrm{CH}_{2} \mathbf{C H}\right)_{3}\right) \\
1.85-1.91 \\
\left(\mathbf{C H}_{2} \mathrm{CH}_{3}\right)\end{array}$ & 2.55 & \\
\hline $\begin{array}{ll}3 & \mathrm{E}\end{array}$ & $\begin{array}{l}5.17 \\
\text { (s) }\end{array}$ & $\begin{array}{l}2.64- \\
2.80\end{array}$ & $\begin{array}{l}2.64- \\
2.80\end{array}$ & $\begin{array}{l}6.32 \\
(d, 7.52)\end{array}$ & $\begin{array}{l}0.86(\mathrm{t}, 7.21) \\
\left.\left(\mathrm{CH}_{2} \mathbf{C H}\right)_{3}\right) \\
1.60-1.62 \\
\left(\mathbf{C H}_{2} \mathrm{CH}_{3}\right)\end{array}$ & $\begin{array}{l}1.15 \\
(\mathrm{t}, 6.93) \\
\left(\mathrm{COCH}_{2} \mathbf{C}\right. \\
\left.\mathbf{H}_{3}\right) \\
2.55^{*}, \\
2.64-2.80 \\
\left(\mathrm{COCH}_{2} \mathrm{C}\right.\end{array}$ & $\begin{array}{ll}5.66(1 \mathrm{H}), & 5.70(2 \mathrm{H}), \\
5.72(1 \mathrm{H}), & 5.86(2 \mathrm{H}), \\
5.90(2 \mathrm{H}), & 6.85(1 \mathrm{H})\end{array}$ \\
\hline
\end{tabular}


RASĀYAN $J$. Chem.

Vol. 10 | No. 1 |25-31 | January - March | 2017

\begin{tabular}{|c|c|c|c|c|c|c|c|}
\hline & & & & & & $\left.\mathrm{H}_{3}\right)$ & \multirow{2}{*}{$\begin{array}{l}6.87(1 \mathrm{H}), 6.90(1 \mathrm{H}), \\
6.94(1 \mathrm{H})\end{array}$} \\
\hline $\mathrm{Z}$ & $\begin{array}{l}6.14 \\
(s)\end{array}$ & $\begin{array}{l}2.64- \\
2.80\end{array}$ & $\begin{array}{l}2.92 \\
(\mathrm{dd}, \\
7.89,1 \\
5.37) \\
2.64- \\
2.80\end{array}$ & $\begin{array}{l}5.47 \\
(\mathrm{~d}, 7.38)\end{array}$ & $\begin{array}{l}0.83(\mathrm{t}, 7.18) \\
\left(\mathrm{CH}_{2} \mathbf{C H}_{3}\right) \\
1.69-1.73 \\
\left(\mathbf{C H}_{2} \mathrm{CH}_{3}\right)\end{array}$ & $\begin{array}{l}1.15(\mathrm{t}, 6.9 \\
3) \\
\left(\mathrm{COCH}_{2} \mathrm{C}\right. \\
\left.\mathbf{H}_{3}\right) \\
2.64-2.80 \\
\left(\mathrm{COCH}{ }_{2} \mathrm{C}\right. \\
\left.\mathrm{H}_{3}\right)\end{array}$ & \\
\hline $\begin{array}{ll}4 & \mathrm{E}\end{array}$ & $\begin{array}{l}5.23 \\
\text { (s) }\end{array}$ & $\begin{array}{l}2.73- \\
2.78\end{array}$ & $\begin{array}{l}2.94- \\
3.07 \\
2.89( \\
d, 15.3 \\
4)\end{array}$ & $\begin{array}{l}6.55 \\
(\mathrm{~d}, 6.95)\end{array}$ & $\begin{array}{l}0.81(\mathrm{t}, 7.31) \\
\left(\mathrm{CH}_{2} \mathbf{C H}_{3}\right) \\
1.81-1.94 \\
\left(\mathbf{C H}_{2} \mathrm{CH}_{3}\right)\end{array}$ & - & \multirow[t]{2}{*}{$\begin{array}{lr}5.89(1 \mathrm{H}), & 5.94- \\
5.97(3 \mathrm{H}), & 6.07- \\
6.09(4 \mathrm{H}), & 7.10(1 \mathrm{H}), \\
7.12(2 \mathrm{H}), & 7.15(1 \mathrm{H}), \\
7.46-7.47, & \\
7.73\left(\mathrm{COC}_{6} \mathrm{H}_{5}\right)\end{array}$} \\
\hline $\mathrm{Z}$ & $\begin{array}{l}6.31 \\
\text { (s) }\end{array}$ & $\begin{array}{l}2.73- \\
2.78\end{array}$ & $\begin{array}{l}2.94- \\
3.07\end{array}$ & $\begin{array}{l}5.48 \\
(\mathrm{~d}, 7.33)\end{array}$ & $\begin{array}{l}1.04(\mathrm{t}, 7.29) \\
\left(\mathrm{CH}_{2} \mathbf{C H}_{3}\right) \\
1.61-1.68 \\
\left(\mathbf{C H}_{2} \mathrm{CH}_{3}\right)\end{array}$ & - & \\
\hline
\end{tabular}

\section{Analysis of Chemical Shifts}

The deshielding effects observed due to $N$-acylation in 1-4 are displayed in Table - 3. The deshielding magnitude observed on $\mathrm{H}(2)$ in the $Z$ isomers (syn $\alpha$ protons) are roughly same as those observed on $\mathrm{H}(6)$ in the $E$ isomers (syn $\alpha$ protons) and the magnitude of deshielding is $\approx 2 \mathrm{ppm}$ in 1-4. The deshielding magnitude of syn $\alpha$ protons higher than the syn $\alpha$ axial protons. Moreover, the deshielding magnitude of anti $\alpha$ protons is also higher $(\approx 1 \mathrm{ppm})$ than the anti $\alpha$ axial protons.

Table - 2: ${ }^{13} \mathrm{C}$ NMR values (ppm) of $N$-acyl-3-ethylpiperidin-4-ones (1-4)

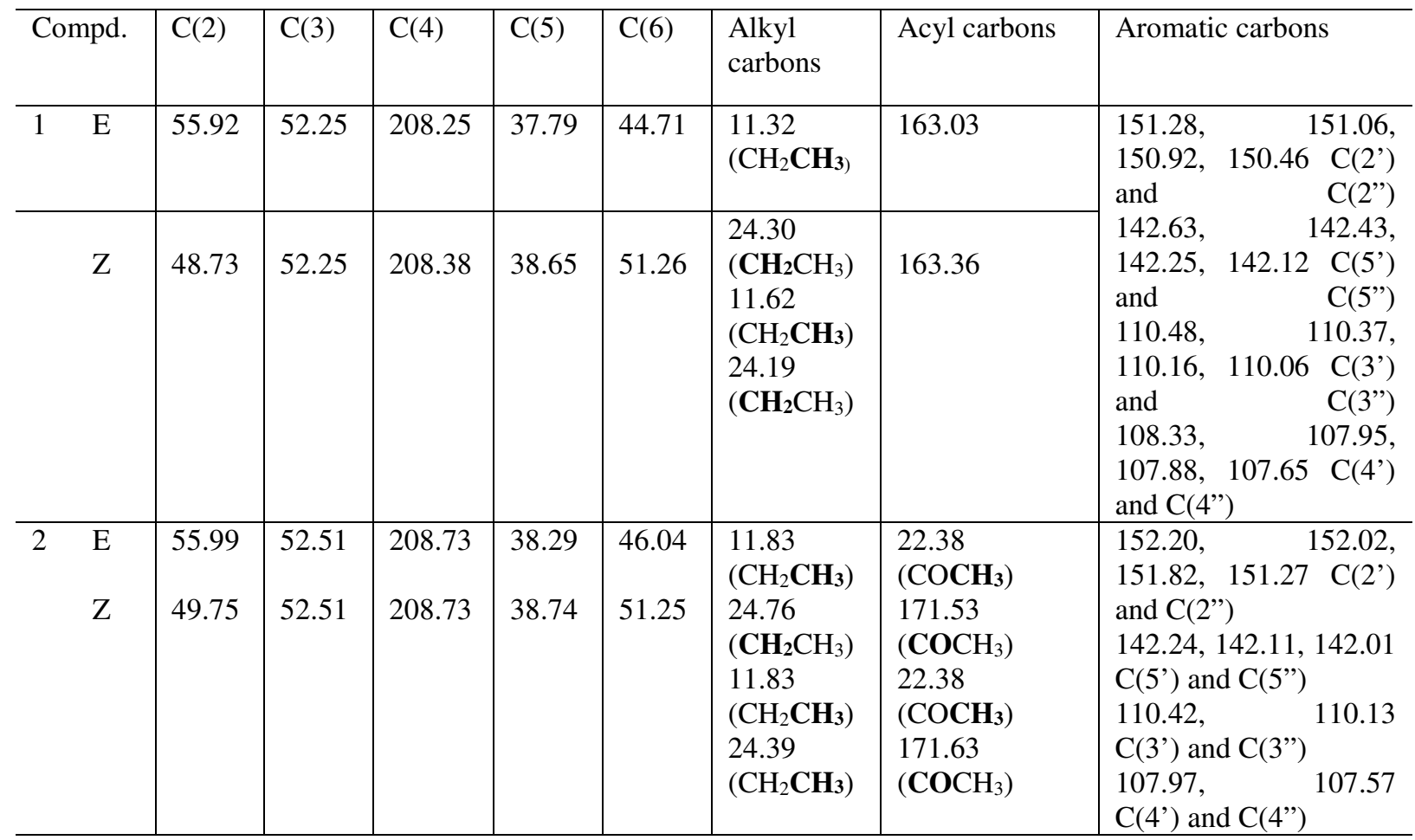


RASĀYAN $J$. Chem.

Vol. 10 | No. 1 |25-31 | January - March | 2017

\begin{tabular}{|c|c|c|c|c|c|c|c|c|c|}
\hline 3 & $\mathrm{E}$ & $\begin{array}{l}54.74 \\
49.94\end{array}$ & $\begin{array}{l}52.56 \\
52.56\end{array}$ & $\begin{array}{l}208.86 \\
208.99\end{array}$ & $\begin{array}{l}38.40 \\
38.76\end{array}$ & $\begin{array}{l}46.25 \\
50.07\end{array}$ & $\begin{array}{l}11.85 \\
\left(\mathrm{CH}_{2} \mathbf{C H}_{3}\right) \\
24.40 \\
\left(\mathbf{C H}_{2} \mathrm{CH}_{3}\right) \\
11.78 \\
\left(\mathrm{CH}_{2} \mathbf{C H}_{3}\right) \\
24.76 \\
\left(\mathrm{CH}_{2} \mathbf{C H}_{3}\right)\end{array}$ & $\begin{array}{l}9.55 \\
\left(\mathrm{COCH}_{2} \mathbf{C H}_{3}\right) \\
26.66 \\
\left(\mathrm{COCH}_{2} \mathrm{CH}_{3}\right) \\
174.45 \\
\left(\mathrm{COCH}_{2} \mathrm{CH}_{3}\right) \\
9.55 \\
\left(\mathrm{COCH}_{2} \mathbf{C H}_{3}\right) \\
26.66 \\
\left(\mathrm{COCH}_{2} \mathrm{CH}_{3}\right) \\
174.58 \\
\left(\mathrm{COCH}_{2} \mathrm{CH}_{3}\right)\end{array}$ & $\begin{array}{lr}152.35, & 152.14, \\
151.85,151.36 & C\left(2^{\prime}\right) \\
\text { and } C\left(2^{\prime \prime}\right) & \\
142.21, & 142.09, \\
141.95,142.85 & C\left(5^{\prime}\right) \\
\text { and } C\left(5^{\prime \prime}\right) & \\
110.43, & \\
110.09,110.31, & 1103\left(3^{\prime}\right) \\
\text { and } C\left(3^{\prime \prime}\right) \\
107.91,107.75,107.49 \\
C\left(4^{\prime}\right) \text { and } C\left(4^{\prime \prime}\right)\end{array}$ \\
\hline 4 & $\mathrm{E}$ & $\begin{array}{l}56.43 \\
50.82\end{array}$ & $\begin{array}{l}53.60 \\
52.77\end{array}$ & $\begin{array}{l}208.75 \\
208.75\end{array}$ & $\begin{array}{l}38.49 \\
39.37\end{array}$ & $\begin{array}{l}46.72 \\
52.61\end{array}$ & $\begin{array}{l}11.73 \\
\left(\mathrm{CH}_{2} \mathbf{C H}_{3}\right) \\
24.25 \\
\left(\mathbf{C H}_{2} \mathrm{CH}_{3}\right) \\
12.03 \\
\left(\mathrm{CH}_{2} \mathbf{C H}_{3}\right) \\
24.68 \\
\left(\mathrm{CH}_{2} \mathbf{C H}_{3}\right)\end{array}$ & $\begin{array}{l}173.00 \\
173.00\end{array}$ & $\begin{array}{l}152.10,151.81,151.10 \\
\mathrm{C}\left(2^{\prime}\right) \text { and } \mathrm{C}\left(2^{\prime \prime}\right) \\
142.90,142.68,142.02 \\
\mathrm{C}\left(5^{\prime}\right) \text { and } \mathrm{C}\left(5^{\prime \prime}\right) \\
135.34, \quad 130.17, \\
128.83,127.35,126.90 \\
\left(\mathrm{COC}_{6} \mathrm{H}_{5}\right) \\
110.49, \\
\mathrm{C}\left(3^{\prime}\right) \text { and } \mathrm{C}\left(3^{\prime \prime}\right) \\
108.54, \\
\mathrm{C}\left(4^{\prime}\right) \text { and } \mathrm{C}\left(4^{\prime \prime}\right)\end{array}$ \\
\hline
\end{tabular}

With a view to determining the ${ }^{13} \mathrm{C}$ substituent parameters of the formyl, acetyl and benzoyl substituents at nitrogen in the six-membered ring compounds, the chemical shifts of $N$-formylpiperidine ${ }^{12}, N$ acetylpiperidine ${ }^{13}$ and $N$-benzoylpiperidine ${ }^{14}$ are compared with that of the parent piperidine and the parameters are displayed in Table-4. It is seen from Table -4 that syn $\alpha$ carbons are shielded to 5-7 ppm due to $N$-acylation. The anti $\alpha$, syn $\beta$, anti $\beta$ and $\gamma$ carbons are also shielded to $1-3 \mathrm{ppm}$.

Table-3: Observed deshielding magnitude (ppm) in $N$-acyl-3-ethylpiperidin-4-one derivatives (1-4)

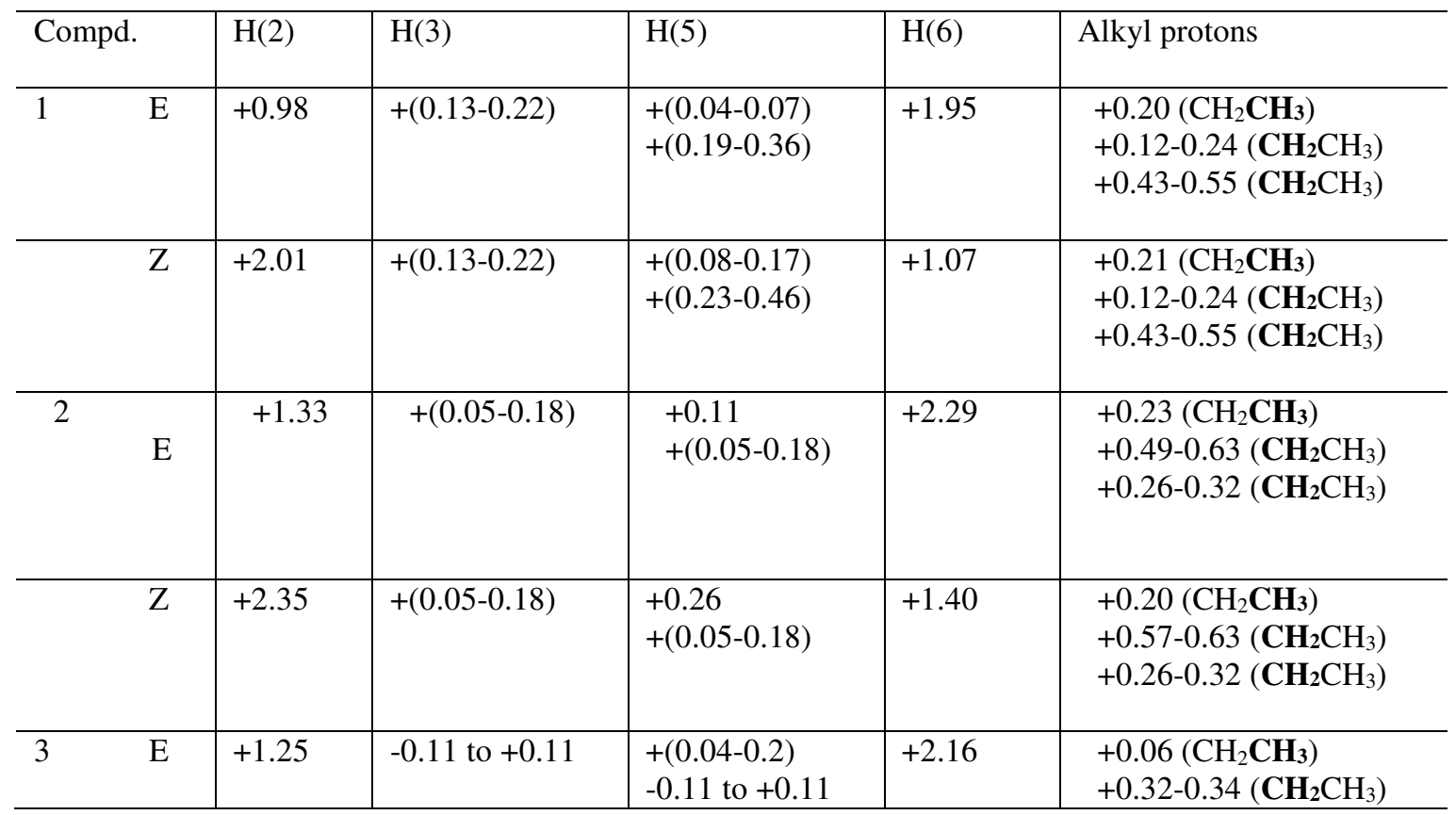


RASĀYAN $J$. Chem.

Vol. 10 | No. 1 |25-31 | January - March | 2017

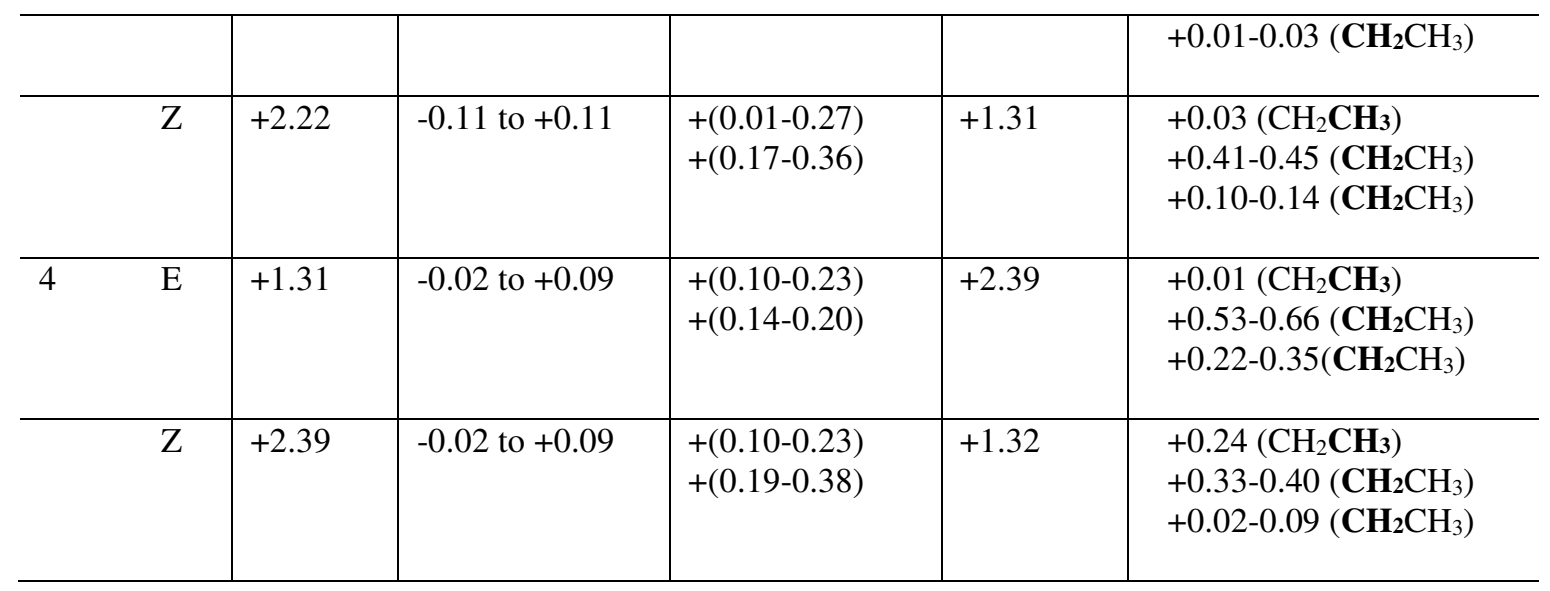

Table-4: Observed shielding magnitude (ppm) of some simple N-acyl piperidines and N-acyl-3-ethylpiperidin-4one derivatives (1-4)

\begin{tabular}{|c|c|c|c|c|c|c|}
\hline \multirow{2}{*}{\multicolumn{2}{|c|}{ Compd. }} & \multicolumn{2}{|l|}{$\alpha$} & \multicolumn{2}{|l|}{$\beta$} & \multirow[t]{2}{*}{$\gamma$} \\
\hline & & syn & anti & syn & anti & \\
\hline \multicolumn{2}{|c|}{ N-Formylpiperidine } & $\begin{array}{l}40.57 \\
(-7.33) \\
\end{array}$ & $\begin{array}{l}46.76 \\
(-1.14) \\
\end{array}$ & $\begin{array}{l}26.66 \\
(-1.24)\end{array}$ & $\begin{array}{l}24.75 \\
(-3.15) \\
\end{array}$ & $\begin{array}{l}25.16 \\
(-1.04) \\
\end{array}$ \\
\hline \multicolumn{2}{|c|}{ N-Acetylpiperidine } & $\begin{array}{l}41.58 \\
(-6.32)\end{array}$ & $\begin{array}{l}46.66 \\
(-1.24)\end{array}$ & $\begin{array}{l}25.29 \\
(-2.61)\end{array}$ & $\begin{array}{l}26.08 \\
(-1.82)\end{array}$ & $\begin{array}{l}24.12 \\
(-2.08)\end{array}$ \\
\hline \multicolumn{2}{|c|}{ N-Benzoylpiperidine } & $\begin{array}{l}42.60 \\
(-5.30) \\
\end{array}$ & $\begin{array}{l}26.11 \\
(-1.79) \\
\end{array}$ & $\begin{array}{l}24.60 \\
(-1.60)\end{array}$ & $\begin{array}{l}26.11 \\
(-1.79)\end{array}$ & $\begin{array}{l}48.30 \\
(+0.40)\end{array}$ \\
\hline \multicolumn{2}{|c|}{ Piperidine } & \multicolumn{2}{|l|}{47.90} & \multicolumn{2}{|l|}{27.90} & 26.20 \\
\hline & $\mathrm{C}(2)$ & $\mathrm{C}(3)$ & $\mathrm{C}(4)$ & $\mathrm{C}(5)$ & $C(6)$ & $\begin{array}{l}\text { Methyl } \\
\text { group }\end{array}$ \\
\hline $\begin{array}{ll}1 & E\end{array}$ & -2.90 & -3.92 & +0.62 & -9.14 & -9.40 & $\begin{array}{l}- \\
0.46(\mathrm{CH} \\
2 \mathrm{CH3}) \\
+6.13(\mathrm{C} \\
\mathrm{H} 2 \mathrm{CH} 3)\end{array}$ \\
\hline $\mathrm{Z}$ & -10.09 & -3.92 & +0.75 & -8.28 & -2.85 & $\begin{array}{l}- \\
0.16(\mathrm{CH} \\
2 \mathbf{C H 3}) \\
+6.02(\mathbf{C} \\
\mathbf{H} 2 \mathrm{CH} 3)\end{array}$ \\
\hline $\mathrm{E}$ & -2.83 & -3.66 & +1.10 & -8.64 & -8.07 & $\begin{array}{l}+0.05(\mathrm{C} \\
\mathrm{H} 2 \mathbf{C H 3}) \\
+6.59(\mathbf{C} \\
\mathbf{H} 2 \mathrm{CH} 3)\end{array}$ \\
\hline $\mathrm{Z}$ & -9.07 & -3.66 & +1.10 & -8.19 & -2.86 & $\begin{array}{l}+0.05(\mathrm{C} \\
\mathrm{H} 2 \mathrm{CH3}) \\
+6.22(\mathbf{C} \\
\mathbf{H} 2 \mathrm{CH} 3)\end{array}$ \\
\hline $\mathrm{E}$ & -4.08 & -3.61 & +1.23 & -8.53 & -7.86 & $\begin{array}{l}- \\
0.07(\mathrm{CH} \\
2 \mathbf{C H 3}) \\
+6.23(\mathbf{C} \\
\mathbf{H} 2 \mathrm{CH} 3)\end{array}$ \\
\hline $\bar{Z}$ & -8.88 & -3.61 & +1.36 & -8.17 & -4.04 & $+0.00(\mathrm{C}$ \\
\hline
\end{tabular}


RASĀYAN J. Chem.

Vol. 10 | No. 1 |25-31 | January - March | 2017

\begin{tabular}{|c|c|c|c|c|c|c|c|}
\hline & & & & & & & $\begin{array}{l}\mathrm{H} 2 \mathbf{C H 3}) \\
+6.59(\mathbf{C} \\
\mathbf{H} 2 \mathrm{CH} 3) \\
\end{array}$ \\
\hline 4 & $E$ & -2.39 & -2.53 & +1.12 & -8.44 & -7.39 & $\begin{array}{l}-\overline{0} \\
0.05(\mathrm{CH} \\
2 \mathbf{C H 3}) \\
+6.08(\mathbf{C} \\
\mathbf{H} 2 \mathrm{CH} 3)\end{array}$ \\
\hline & $\mathrm{Z}$ & -8.00 & -3.40 & +1.12 & -7.56 & -1.50 & $\begin{array}{l}+0.25(\mathrm{C} \\
\mathrm{H} 2 \mathrm{CH} 3) \\
+6.51(\mathrm{C} \\
\mathbf{H} 2 \mathrm{CH} 3)\end{array}$ \\
\hline
\end{tabular}

\section{REFERENCES}

1. T. Taniguchi and K. Ogasawara, Org. Lett., 2(20), 3193(2000).

2. A.R. Katritzky, H.H. Odens, S. Zhang, J. Org. Chem., 66, 6792(2001).

3. H.I. El-Subbagh, S.M. Abu-Zaid, M.A. Mahran, F.A. Badria, A.M. Al-obaid, J. Med. Chem., 43, 2915(2000).

4. B.R. Jerom and K.H. Spencer, Eur. Pat. Appl. 1988, EP 277794.

5. R. Venkateswaramoorthi and K. Krishnasamy, International Journal of Recent Scientific Research, 4(1), 45 (2013)

6. C.R. Noller and V. Baliah , J. Am. Chem. Soc., 70 (11), 3853(1948)

7. K. Selvaraju and A. Kishore Babu, International Journal of Chemtech Research, 8(7), 363 (2015).

8. S. Sivakumar and A. Manimekalai, Spectrochimica Acta part A, Molecular and Biomolecular Spectroscopy, 75(1), 113 (2010).

9. R. Jayaraman, J C. Thenmozhiyal, R. Murugadoss and M. Venkatraj, Indian J. Chem., 38B, 325 (1999).

10. R. Krishnakumar and M. Krishnapillay, Indian J. Hetero. Chem., 2, 379 (1993).

11. Manimekalai, J. Jayabharathi, V. M. Porselvi, N. Prabha, Indian J. Chem., 46B, 681 (2007).

12. C. J. Pouchert, The Aldrich Library of NMR Spectra, Edition II, A (2591-86-8), B (768-66-1).

13. B. Mario Pinto, T.Bruce Grindley and W. A. Szarek, Magn. Reson. Chem., 24, 323 (1986).

14. C. W. Fong and H. G. Grant, Aust. J. Chem., 34, 2307(1981).

[RJC-1544/2016] 